\title{
Multigroup cross section library for GFR2400
}

\author{
Štefan Čerba ${ }^{a}$, Branislav Vrban, Jakub Lüley, Ján Haščík, and Vladimír Nečas \\ Slovak University of Technology in Bratislava, Faculty of Electrical Engineering and Information Technology, Institute of Nuclear and \\ Physical Engineering, Ilkovičova 3, 81219 Bratislava, Slovakia
}

\begin{abstract}
In this paper the development and optimization of the SBJ_E71 multigroup cross section library for GFR2400 applications is discussed. A cross section processing scheme, merging Monte Carlo and deterministic codes, was developed. Several fine and coarse group structures and two weighting flux options were analysed through 18 benchmark experiments selected from the handbook of ICSBEP and based on performed similarity assessments. The performance of the collapsed version of the SBJ_E71 library was compared with MCNP5 CE ENDF/B VII.1 and the Korean KAFAX-E70 library. The comparison was made based on integral parameters of calculations performed on full core homogenous models.
\end{abstract}

\section{Introduction}

The progress in computer technology in the $21^{\text {th }}$ century gives strong support to the development of modern Monte Carlo codes. Unfortunately, their results are burdened with statistical errors. Moreover, due to CE XS libraries and complex geometry structures Monte Carlo simulations are costly. For these reasons certain reactor applications require effective deterministic approaches, which imply the development of multi-group cross section (XS) libraries. There exist several multi-group XS libraries available for fast reactor calculations; however, each of them carries a unique fingerprint of a system, for which it was developed and optimized. The best way to optimize a $\mathrm{XS}$ library is to use as much experimental data as possible, this could be however impossible for systems that have never been built, like the GEN IV Gas-cooled Fast Reactor [1]. The analysts of this reactor are facing a difficult task, to design a reactor without having experimental background. One of the possible ways could be to find a balance between deterministic and stochastic calculation tools and to utilize experimental data of similar fast systems. The first necessary step is to develop and to optimize a multigroup XS library appropriate for deterministic GFR2400 analyses.

\section{Description of the GFR2400 core}

The Gas-cooled Fast reactor is one of the GEN IV nuclear reactors selected by the GIF for further development. GFR2400 is a large scale power unit with a thermal power of $2400 \mathrm{MWth}$. The cross-sectional view of the reactor core is shown in Fig. 1. The reactor core consists of two zones, the inner fuel core (IF) and outer fuel core (OF). The inner and outer fuel cores consist of 264 and 252 (U,Pu,Am)C fuel assemblies with $\mathrm{SiC}-\mathrm{SiC}_{\mathrm{fib}}$ cladding and W14Re-Re refractory liner. The volumetric content of $\mathrm{Pu}$ isotopes in heavy metal in the IF and OF fuel

a e-mail: srefan.cerba@stuba.sk assemblies reach $14.2 \%$, and $17.6 \%$. The core fuel region is surrounded by fiver rings of $\mathrm{Zr}_{3} \mathrm{Si}_{2}$ reflector assemblies in the radial direction and by a $1 \mathrm{~m}$ high axial reflector below and above the fission gas plena. The reactivity of the GFR2400 core is controlled through two systems of control rods, CSD and DSD assemblies. Both systems accommodate $\mathrm{B}_{4} \mathrm{C}$ absorbers with $90 \%$ weight content of ${ }^{10} \mathrm{~B}$ isotope [1]. The fuel loading patter of the GFR2400 reactor is shown in Fig. 2.

\section{The cross section processing scheme}

As it was mentioned, deterministic calculations of the GFR2400 reactor require multigroup XS libraries optimized for a given purpose. The XS preparation scheme of our SBJ_E71 XS library, developed for GFR2400, is shown in Fig. 3.

The scheme was created based on previous experience of the authors published in [2] and merges evaluated data and deterministic and stochastic calculation tools. It starts with the processing of ENDF/B-VII.1 [3] evaluated data (1). For demonstrational purposes, only one evaluated data source was used, but the XS preparation scheme is not limited to only ENDF/B-VII.1. The scheme is available for any ENDF6 format nuclear data. The processing of evaluated data is based on the selected list of nuclides (2) and temperatures (3) in the NJOY99 [4] code (4). The produced CE XS library (5) and the material composition (6) are used in the MCNP5 [5] code (7) to calculate the core averaged neutron spectrum and to transform it to multigroup NJOY99 weighting flux. The weight flux (8), the ENDF/B-VII.1 evaluated data and the background XSs (9) are then processed in NJOY99 (10) to produce fine group MATXS cross-section libraries (11). These cross-sections are transformed to effective region-wise macroscopic XS data using TRANSX [6] (12) and stored in the ISOTXS library (13). In order to accelerate the deterministic full core calculations group collapsing can be performed in TRANSX (17), based on the RZFLUX (15) region-wise neutron flux obtained from RZ transport

(C) The Authors, published by EDP Sciences. This is an Open Access article distributed under the terms of the Creative Commons Attribution License 4.0 (http://creativecommons.org/licenses/by/4.0/). 


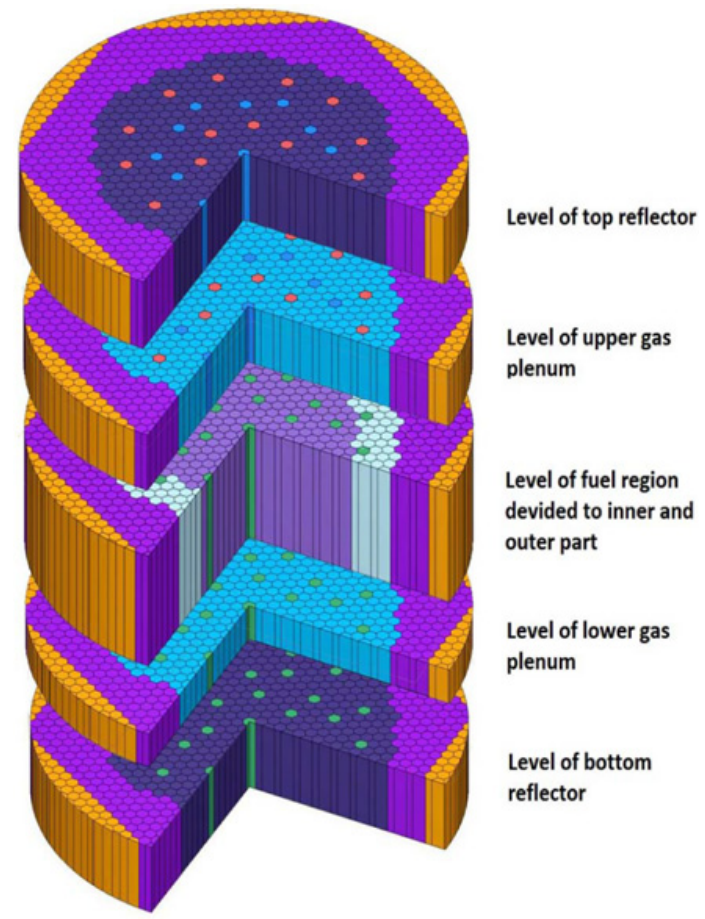

Figure 1. Cross sectional view of the GFR2400 reactor.

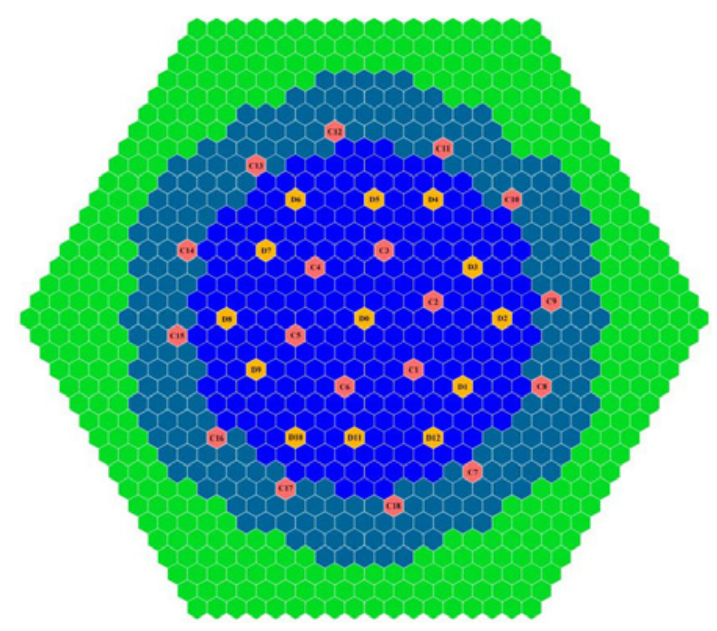

Figure 2. Fuel loading pattern of the pin type GFR2400 core.

calculation in PARTISN [7] (15). Than coarse group ISOTXS library (18) is created and used in DIF3D [8] (19) to perform fast and accurate core calculations (20).

The precision of XS processing based on the Bondarenko method is mainly influenced by the background cross-section data, the weighting flux and the energy group structure of the library. The background cross sections are isotope and energy dependent parameters. Since research of these data falls outside our scope, in our approach the background XSs were adopted from the Korean KAFAXE70 [9] XS library. To determine the influence of energy group structures on the quality of multigroup XS data, 4 energy group structures (LANL 80 g, LANL $187 \mathrm{~g}$, SANDII $500 \mathrm{~g}$ and SAND-II $620 \mathrm{~g}$ ) were investigated. The $80 \mathrm{~g}$, $187 \mathrm{~g}$ and $620 \mathrm{~g}$ are standard NJOY99 energy structures. The $500 \mathrm{~g}$ library is a modified version of the SAND-II $620 \mathrm{~g}$, where the thermal energy range has been merged to one energy group. In case of each group structure

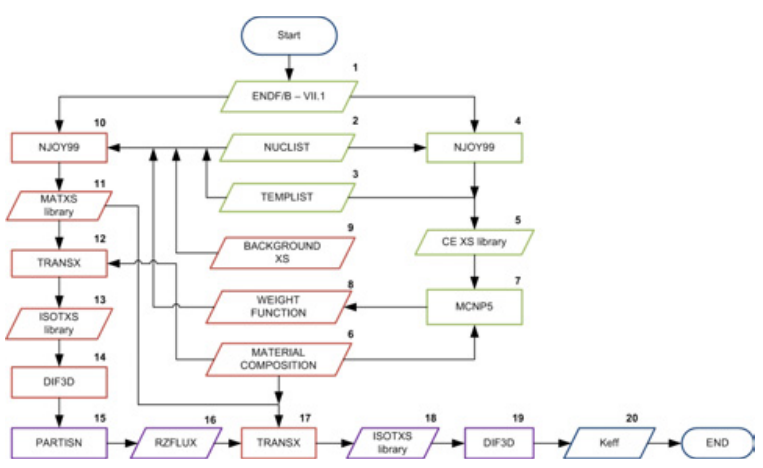

Figure 3. The cross section processing scheme.

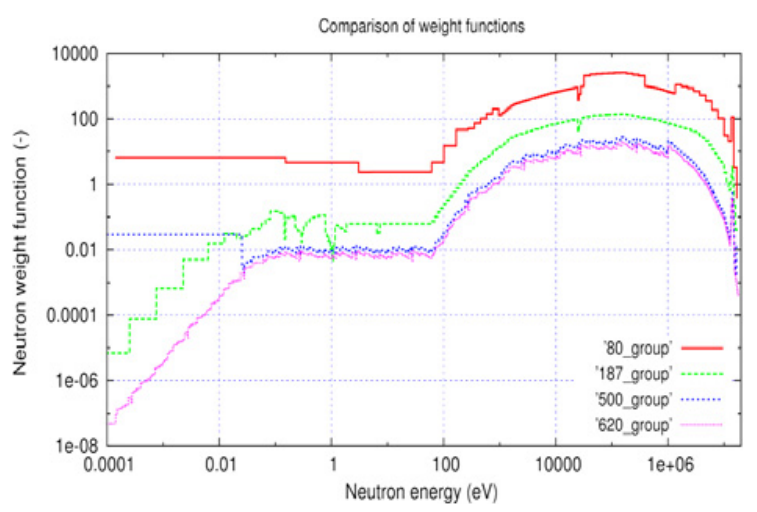

Figure 4. Representation of the IWT8 weighting flux.

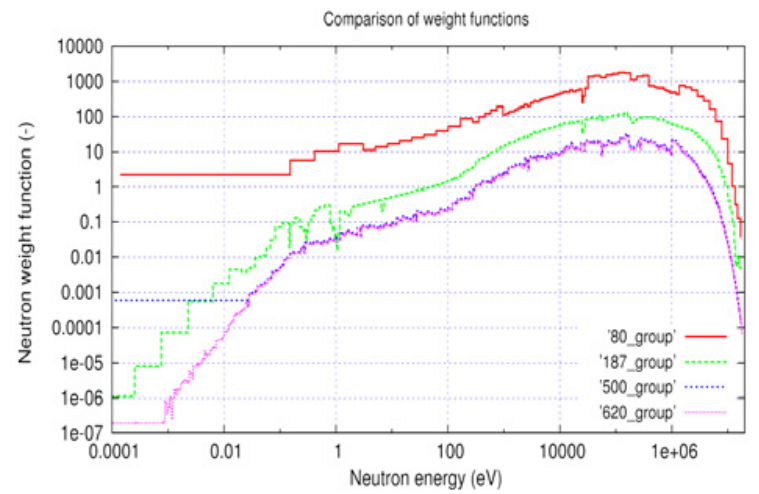

Figure 5. Representation of the core averaged GFR2400 neutron spectrum.

two weighting functions were used. In the presented analysis the GFR2400 neutron spectrum weight function was compared with the standard NJOY99 "IWT8" option, which represents a typical LMFR neutron spectrum. The IWT8 weighting flux is shown in Fig. 4 and the GFR2400 average neutron spectrum in Fig. 5.

\section{Analyses and results}

\subsection{Benchmarking}

The bias of the created multigroup XS libraries was evaluated through benchmark analyses. Based on the 2015 edition of the handbook of the ICSBEP project [10], recommendations of WPEC SG33 [11] and results of a similarity assessments performed in SCALE6 18 benchmarks were selected and prepared in a form of PARTISN input files. For each benchmark, 4 energy group structures and 2 weighting options were investigated. The 


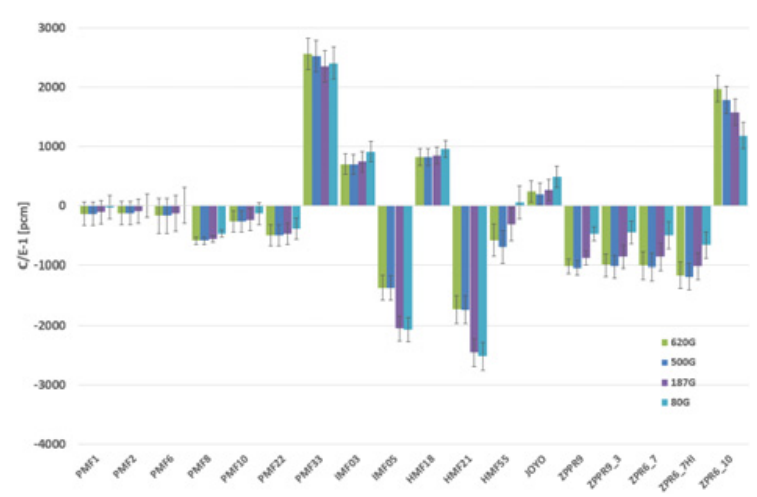

Figure 6. Benchmark results for various group structures.

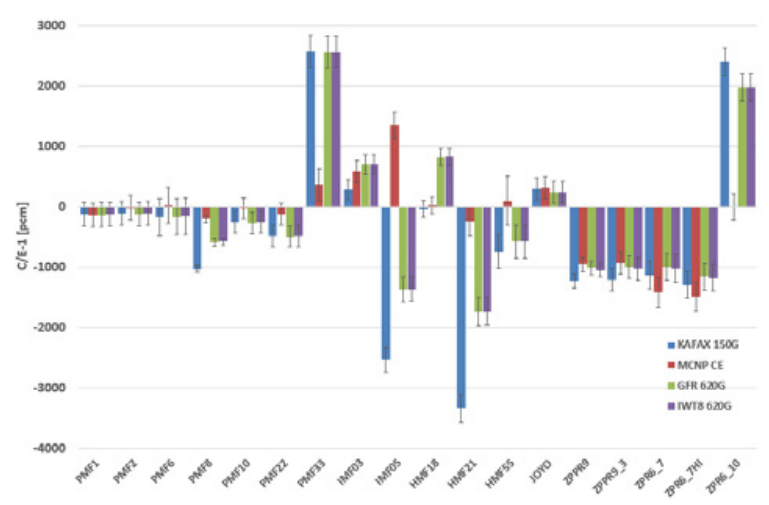

Figure 7. Comparison with KAFAX and MCNP5.

calculations were performed with the fine group versions of the SBJ_E71 XS libraries and the results were compared between calculation cases as well as with deterministic calculations using the $150 \mathrm{~g}$ KAFAX-E70 library and with CE MCNP5 results using the C/E-1 parameter. The benchmark results presented in Fig. 6 show that the use of slightly different weighing fluxes has only minor effect $(1.5-29 \mathrm{pcm})$ on the $\mathrm{k}_{\mathrm{eff}}$ results. The influence of group structure was also not the main source of $\mathrm{k}_{\mathrm{eff}}$ deviation. The bias of the SBJ_E71 library to the experiments depends mainly on the complexity of geometry models and required simplifications for deterministic codes. The comparison of the SBJE71 library with KAFAX-E70 and MCNP5 CE results is shown in Fig. 7.

\subsection{Application for GFR2400}

Since the benchmark cases showed promising results it was justified to use our XS library for GFR2400 core calculations. To complete the calculation scheme two commonly used coarse group structures were adopted, the $25 \mathrm{~g}$ and $33 \mathrm{~g}$ structures. These structures were used for collapsing the IWT8 and GFR versions of the 80 , 187,500 and 620 group libraries. The $25 \mathrm{~g}$ and $33 \mathrm{~g}$ libraries were used for HEX-Z full core calculations in DIF3D. The results were compared with $150 \mathrm{~g}$ KAFAXE70 and MCNP5 CE calculations, performed on the same homogenous model. The results (see Fig. 8) show significant deviations between the $80 \mathrm{~g}$ and the remaining group structures. The 80 to 25 and 80 to 33 structures overestimated the excess reactivity of the system by $301-310 \mathrm{pcm}$, while the remaining structures underestimated, compared to MCNP5. An interesting finding is that the reactivity deviation between the 620 to 33 and 187 to

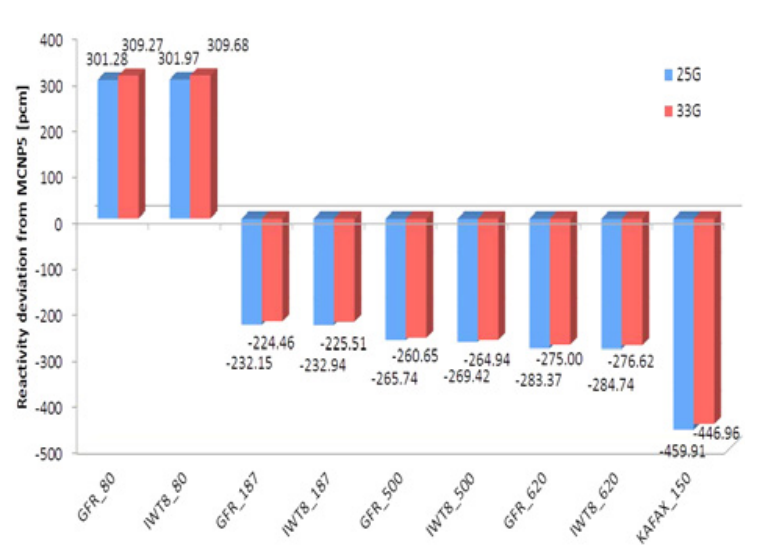

Figure 8. Results of the GFR2400 core calculation.

33 libraries was only $50 \mathrm{pcm}$, what allows us to use the $187 \mathrm{~g}$ structure for fine group core calculations. It was also found that the average difference between the $25 \mathrm{~g}$ and $33 \mathrm{~g}$ coarse group structure is only $8 \mathrm{pcm}$.

\section{Conclusion}

Our optimized SBJ_E71 XS multigroup library was prepared based on ENDF/B-VII.1 evaluated data and KAFAX-E70 background XSs. It was prepared in various fine and coarse group structures and two weighting flux options were used for each version. The fine group versions were tested through 18 benchmark experiments. The benchmarks showed promising $\mathrm{C} / \mathrm{E}-1 \mathrm{k}$ eff results in comparison with the KAFAX-E70 library, and the MCNP5 CE calculation. In case of simple systems (PU MET FAST cases) the reactivity deviation was less than $300 \mathrm{pcm}$. In case of complex systems, such as JOYO and ZPPR9, all XS multigroup libraries were characterized by approximately $1000 \mathrm{pcm}$ bias, caused by the homogenization effect. This effect can be seen also from the results of MCNP5 calculations performed on the simplified benchmark models. The core calculations performed on the 3D HEX$\mathrm{Z}$ model of GFR2400 in DIF3D pointed out, that after group collapsing the effect of fine group weighting flux option in NJOY99 becomes negligible. However; this weighting flux plays an important role in the generation of the RZFLUX file, which is used for group collapsing. Except the $80 \mathrm{~g}$ structure, the remaining 3 energy structures could be used for fine group calculations with very similar computational bias, however the lower calculation time makes the $187 \mathrm{~g}$ library more suitable. The performed calculations showed that the precision of the SBJ_E71 library is comparable with KAFAX-E70, but SBJ_E71 is more suitable for GFR2400 calculations. As a result of the presented optimization study the $187 \mathrm{~g}$ fine group, $33 \mathrm{~g}$ coarse group structure and the GFR2400 weighting flux were selected as basic processing options for future uses of the SBJ_E71 library. The most important finding of this analysis is that using the SBJ_E71 library in $25 \mathrm{~g}$ or $33 \mathrm{~g}$ structure reliable results can be obtained in approximately 30 s calculation time, while the same CE MCNP5 analysis requires 24 hours of execution on a cluster system. In order to better assess the precision and usability of the SBJ_E71 library, more benchmark experiments will have to be evaluated, and the GFR2400 calculations will have to 
be compared based on core-wide distributions and groupwise collision rates, not only $\mathrm{k}_{\text {eff }}$.

This work was financially supported by grant of the Slovak Research and Development Agency No. APVV-0123-12 and grant scheme VEGA 1/0796/13.

\section{References}

[1] GFR 2400 MWth pin core at start of GoFastR (GoFastR, 2009)

[2] Š. Čerba et al., Development of multigroup neutron cross section library for fast reactor calculations (Proceedings of the 22nd international conference on applied physichs of condensed matter, pp. 29-36, ISBN: 978-961-6702-39-3 (2016)

[3] M.B Chadwick et al., ENDF/B-VII.1 Nuclear Data for Science and Technology: Cross Sections, Covariances, Fission Product Yields and Decay Data, Nuclear Data Sheets, 112(2), 2887-2996 (2011)

[4] R.E. MacFarlane et al., NJOY99: Data Processing System of Evaluated Nuclear Data Files ENDF Format (Los Alamos National Laboratory, 2000)
[5] LANL, MCNP - A General N - Particle Transport Code (LANL, 2003)

[6] R.E. MacFarlane, TRANSX-CTR: A Code for Interfacing MATXS Cross-Section Libraries to Nuclear Transport Codes for Fusion Systems Analysis (LANL, 1984)

[7] ORNL, PARTISN: Multi-Dimensional, TimeIndependent or Time-Dependent, Multigroup, Discrete Ordinates Transport Code System (RSIC, 2009)

[8] ORNL, DIF3D: Code System Using Variational Nodal Methods and Finite Difference Methods to Solve Neutron Diffusion and Transport Theory Problems (RSIC, 2011)

[9] D.H. Kim, C.S. Gil, Y.O. Lee, ZZ KAFAX-E70, 150 and 12 Groups Cross Section Library in MATXS Format based on ENDF/B-VII.O for Fast Reactors (KAERI, Daejeon, 2008)

[10] OECD NEA, International Handbook of Evaluated Criticality Safety Benchmark Experiments (OECD, Paris, 2007)

[11] OECD NEA, Methods and Issues for the Combined Use of Integral Experiments and Covariance Data (OECD, Paris, 2013) 